\title{
Diversidad de aves en fragmentos de bosque seco tropical en paisajes ganaderos del Departamento de Córdoba, Colombia
}

\author{
José A. Vergara Paternina ${ }^{1}$, Jesús Ballesteros Correa $^{2}$, Carlos González Charrasquiel ${ }^{3}$ \\ \& Juan C. Linares Arias ${ }^{4}$ \\ 1. Programa de Ganadería y Manejo del Medio Ambiente (GAMMA), Centro Agronómico Tropical de Investigación y \\ Enseñanza (CATIE), Turrialba, Costa Rica; jvergara@catie.ac.cr \\ 2. Grupo de Investigación Biodiversidad UNIcórdoba, Universidad de Córdoba, Montería, Colombia; \\ jballescor@yahoo.com \\ 3. Grupo de Investigación Biodiversidad UNIcórdoba, Universidad de Córdoba, Montería, Colombia; \\ gonzalez.charrasquiel@gmail.com \\ 4. Grupo de Investigación Biodiversidad UNIcórdoba, Universidad de Córdoba, Montería, Colombia; \\ jlinarias@correo.unicordoba.ed.co
}

\section{Recibido 21-II-2017. Corregido 20-VII-2017. Aceptado 21-VIII-2017.}

\begin{abstract}
Bird diversity in tropical dry forest fragments in cattle landscapes of the Department of Córdoba, Colombia. In Colombia the tropical dry forest is one of the most threatened and least known ecosystems; these have been impacted by different transformation processes and habitat fragmentation, which have affected bird conditions and survival. This research evaluated the influence of landscape elements (forest and pastures with different tree covers) on bird diversity in the tropical dry forest of Córdoba, Colombia. The study was developed within an extensive livestock landscape to characterize the avifauna in four habitat types formed by fragments of secondary forests with different tree cover: secondary forest, pastures with high tree cover, pastures with low tree cover and only pastures. The influence of tree cover on bird diversity was evaluated with monthly samplings in $200 \mathrm{~m}$ long transects (per habitat), with two observation intervals (6:00 - 9:00 and 15:00-18:00), from September 2011 to May 2012. A total of 6667 individuals belonging to 180 species and 48 families of birds were registered. The most abundant species were Columbina talpacoti (5.37\%), Brotogeris jugularis (5.14 \%), Tyrannus melancholicus (4.81\%) and Bubulcus ibis $(4.80 \%)$. The most abundant family was Tyrannidae (16.4\%), followed by Psitacidae (9.0\%) and Columbidae (8.2 \%). The family with the highest number of species was Tyrannidae with 28 species, followed by Accipitridae and Icteridae with 10 species each. The greatest species richness was present in the secondary forest habitat, followed by the pastures with high tree cover, and pastures with low cover. The lowest abundance and species richness was found in pastures without tree cover with significant differences between the habitats (Fisher, $\mathrm{p}<0.05$ ). According to the Shannon index, the diversity of birds was higher in the secondary forests. In contrast, the lowest diversity was found in pastures without tree cover. The greatest species richness occurred in the months of October and November. In the rest of the sampling period, the number of recorded species and individuals showed some stability. The importance of trees presence in extensive livestock areas was evidenced because they help to contain an important diversity of bird species, and they also generate connectivity among fragments of secondary forests in the cattle landscape. Rev. Biol. Trop. 65 (4): 1625-1634. Epub 2017 December 01.
\end{abstract}

Key words: birds, Caribbean region, biodiversity, silvopastoral systems, cattle ranches.

El bosque seco tropical constituye uno de los ecosistemas más amenazados en el Neotrópico. En Colombia, este tipo de bosque es uno de los ecosistemas más degradados, fragmentados y menos conocidos, principalmente en la región Caribe, donde se encuentra la mayor cobertura original y en los cuales se conoce muy poco sobre la biodiversidad 
presente (Gillespie \& Walter, 2001; Pizano, Cabrera, \& Garcia, 2014; Rodríguez, Banda-R, Reyes, \& Estupiñán, 2012).

La avifauna del bosque seco tropical constituye una mezcla de pocas especies de zonas áridas y húmedas con alta especificidad razón por la cual posee un número reducido de especies (Gillespie \& Walter, 2001). Este hábitat es considerado, para las comunidades de aves, como un ecosistema de transición dentro de un gradiente, convirtiéndose así en un punto clave para el endemismo de especies (Gomez \& Robinson, 2014). La información actualizada de la dinámica de los ensamblajes de comunidades de aves en los distintos remanentes de bosque seco, aportaría conocimiento de las especies que aún se conservan en el Caribe colombiano y sobre cómo estas son afectadas por la falta de conectividad estructural del paisaje. El mosaico de estos paisajes está altamente dominado por áreas dedicadas a la ganadería extensiva y potreros con variadas densidades de árboles dispersos, cercas vivas poco complejas, plantaciones forestales y cultivos agrícolas, adyacentes a pequeños fragmentos de bosque. Estos usos de suelo constituyen los elementos más frecuentes en el paisaje en el departamento de Córdoba, y de casi todo el Caribe colombiano. En ellos aún se refugian muchas especies de aves, desconociéndose su estado actual de conservación, patrones de distribución espacial y temporal, así como el uso de los recursos disponibles en dichos hábitats modificados por las actividades antrópicas.

Trabajos previos han demostrado que la composición de la avifauna en el departamento de Córdoba incluye cerca de 504 especies (Ballesteros, Vergara, \& Linares, 2015), y representa cerca el $27 \%$ de las especies colombianas (Donegan, McMullan, Quevedo, \& Salaman, 2013). Sin embargo, se desconoce cómo los procesos de transformación de hábitat afectan las condiciones y supervivencia de la avifauna local, situación que puede ser aún más crítica en el bosque seco tropical (Cárdenas, Harvey, Ibrahim, \& Finegan, 2003; Schroth et al., 2004; Vilchez et al., 2014).
Actualmente, no hay disponibles publicaciones que describan la diversidad de especies de aves presentes en los bosques secos del Departamento de Córdoba. Atendiendo esta problemática, este trabajo tuvo como objetivo evaluar la influencia de los elementos del paisaje (bosques y potreros de producción ganadera con diferentes densidades de árboles) en la distribución espacial y temporal de la riqueza y abundancia de aves, en el bosque seco tropical del Departamento de Córdoba, Colombia.

\section{MATERIALES Y MÉTODOS}

Área de estudio: Este estudio se realizó en la región Caribe de Colombia, en el Departamento de Córdoba, situado al noroccidente del país entre los $09^{\circ} 26^{\prime}$ - $07^{\circ} 22^{\prime \prime} \mathrm{N} \&$ $74^{\circ} 47^{\prime}-76^{\circ} 30^{\prime} \mathrm{W}$, cuenta con una superficie de $23980 \mathrm{~km}^{2}$ que corresponde al $2.1 \%$ del territorio de Colombia. Esta región posee un clima cálido tropical con temperatura promedio de $28{ }^{\circ} \mathrm{C}$, una precipitación promedio anual de $1300 \mathrm{~mm} / \mathrm{año}$ en distribución unimodal, con una época seca de diciembre a marzo, y una época de lluvias de abril a noviembre. Se seleccionaron cuatro localidades en fincas ganaderas con fragmentos de bosque seco tropical asociados a potreros de producción de ganadería extensiva. En todas las localidades se identificaron cuatro tipos de hábitats que corresponden a: bosque seco tropical secundario (BS), este correspondía a parches de bosque con edades entre los 30 y 60 años sin intervención intensiva; potrero con alta cobertura de árboles (PAC), potreros con baja cobertura de árboles (PBC) y potreros sin árboles (Po). La clasificación para los tipos de hábitats se realizó con base en la clasificación de usos de suelo de Murgueitio et al. (2003), donde los PAC corresponden a potreros con más de 30 árboles ha ${ }^{-1}$ y que tienen un dap > a $5 \mathrm{~cm}$ y $2 \mathrm{~m}$ de altura, los PBC corresponden a potreros con menos de 30 árboles $\mathrm{ha}^{-1}$ y que tienen un dap > a $5 \mathrm{~cm}$ y $2 \mathrm{~m}$ de altura.

Muestreos de aves: Durante todo el estudio un solo observador realizó censos en 
transectos en los cuatro tipos hábitats presentes en el paisaje, con el fin de medir la riqueza y abundancia de las aves presentes. En cada uno de los hábitats se establecieron dos transectos de $200 \mathrm{~m}$ de longitud con ancho variable, estos estuvieron separados entre sí por una distancia de más de $200 \mathrm{~m}$ y recorridos en 25 minutos, registrando todas las aves detectadas de manera visual o auditiva (Buckland, Anderson, Burnham, \& Laake, 2005; Ojasti, 2000; Ralph et al., 1996). Cada uno de los hábitats estuvo representado en las cuatro localidades trabajadas, teniendo al final 32 transectos de observación en todo el periodo de muestreo. Los muestreos se realizaron durante nueve meses en el periodo comprendido desde septiembre 2011 hasta mayo 2012; en cada tipo de hábitat se realizaron muestreos mensuales con un lapso de tiempo de dos semanas entre muestreos y con una repetición por cada mes de muestreo. Cada transecto fue censado en las horas de la mañana de 06:00-09:00 y en la tarde de 15:00-18:00. Se usaron para las observaciones binoculares Brunton Echo 10x42 y cámara fotográfica de referencia Canon Powershot SX30. La identificación de las especies se realizó en campo con guías ilustradas de identificación de Hilty y Brown (1986) y Restall, Rodner y Lentino, (2006).

Se evaluó la representatividad de los muestreos mediante curvas de acumulación de especies usando el estimador de Chao 1 para las especies esperadas en el estudio (Chao, 1984; Krebs, 1999; Magurran, 2004). Con las variables medidas de riqueza y abundancia se realizaron comparaciones estadísticas entre los diferentes tipos de hábitats, usando modelos lineales generalizados y mixtos con comparaciones LSD Fisher y nivel de significancia del $95 \%$, para esto se comprobaron los supuestos de normalidad y heterogeneidad de varianza en los datos. Se calcularon índices de diversidad y dominancia para cada tipo de hábitat (Hill \& Gauch, 1980; Legendre \& Legendre, 1998; Oksanen, 2013; Oksanen et al., 2009). Se realizó un análisis de correspondencia sin tendencia (DCA) con el fin de evaluar la influencia de los hábitats en la distribución de las especies según su presencia y abundancia. Este análisis permite tener una ordenación espacial de las especies en función de los hábitats estudiados. Todos los análisis de datos fueron realizados con los softwares EstimateS 9.1.0 (Colwell, 2013), Infostat versión 2014 (Di Rienzo et al., 2014) y Qeco (Di Rienzo, Casanoves, Pla, Vilchez, \& Di Rienzo, 2010).

\section{RESULTADOS}

Diversidad de aves: Se registraron para todo el estudio 6667 individuos y 180 especies correspondientes a 48 familias. La representatividad de los muestreos según el estimador de Chao 1 para las especies esperadas en el estudio se obtuvo en un $98.1 \%$ para el bosque secundario, $85.9 \%$ para el potrero con alta densidad de árboles, $94.3 \%$ para el potrero con baja densidad de árboles y $87.4 \%$ para el potrero sin árboles. El número de especies de aves fue mayor a medida que aumentó la complejidad de la estructura de la vegetación en los hábitats estudiados, reflejándose en el mayor número de especies acumuladas en las curvas de la gráfica (Fig. 1). La especie más abundante registrada fue Columbina talpacoti con el $5.37 \%$ del total de individuos, seguida de Brotogeris jugularis (5.14\%), Tyrannus melancholicus (4.81\%) y Bubulcus ibis (4.80\%). La familia más abundante fue Tyrannidae con $16.4 \%$ de los individuos registrados, seguida de Psitacidae con $9.0 \%$ y Columbidae con $8.2 \%$. La familia que presentó mayor número de especies reportadas fue Tyrannidae con 28 especies, seguida de Accipitridae e Icteridae con 10 especies cada una.

El hábitat en el que se registró mayor riqueza de especies fue $\mathrm{BS}$ con 135 especies que correspondió al $70 \%$ de las especies registradas, seguido de PAC con 116 especies (64\%), PBC con 101 especies (56\%) y Po con 63 especies $(35 \%)$. Esta riqueza presentó diferencias significativas entre los cuatro tipos de hábitat (LSD Fisher, p=0.002) (Fig. 2). En riqueza de especies el BS sólo presentó diferencias significativas con Po, mientras que entre los demás hábitats que presentan cobertura de 

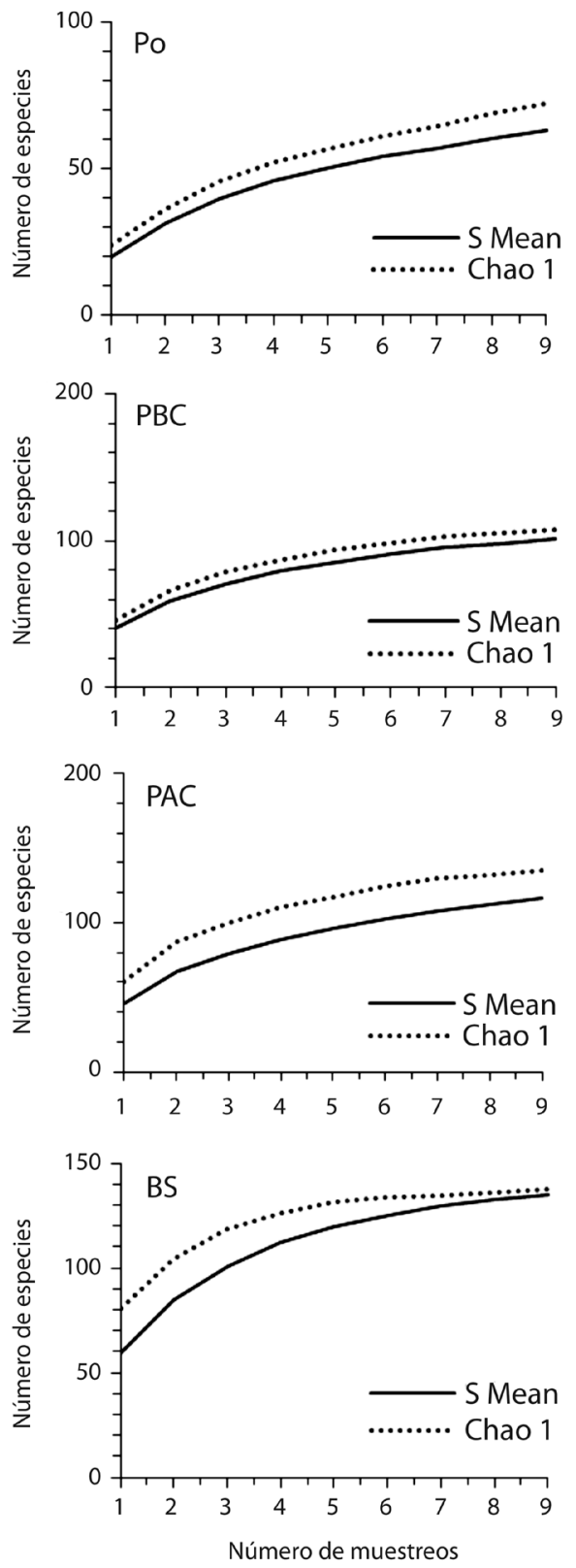

Fig. 1. Curva de acumulación de especies de la avifauna registrada en cuatro hábitats en paisajes ganaderos del Departamento de Córdoba, Colombia. Bosque secundario (BS), potrero con alta cobertura de árboles (PAC), potrero con baja cobertura de árboles (PBC), potrero sin árboles (Po).

Fig. 1. Accumulation curve for birds from four habitats in cattle landscapes of the Department of Córdoba, Colombia. Secondary forest (BS), pasture with high tree cover (PAC), pasture with low tree cover (PBC), treeless pasture (Po), richness estimator Chao 1 (Chao 1).

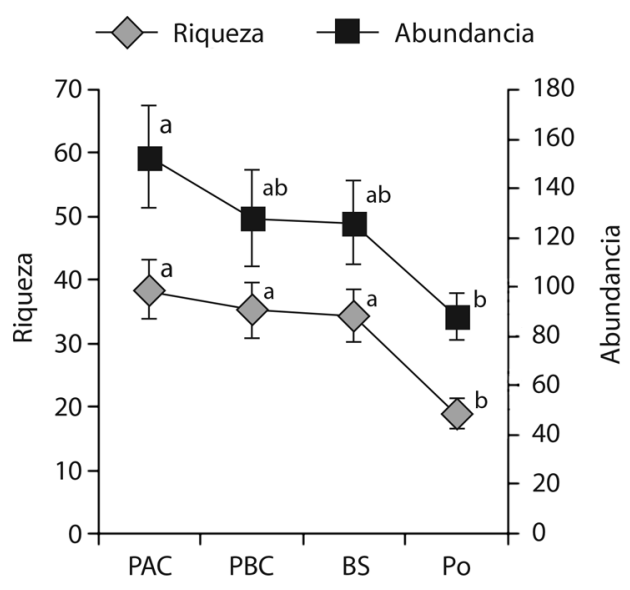

Fig. 2. Comparación de la riqueza y abundancia de aves en cuatro hábitats en paisajes ganaderos del Departamento de Córdoba, Colombia. Letras iguales indican que no hay diferencias significativas.

Fig. 2. Comparison the richness and abundance of bird species in four habitats in cattle landscapes of the Department of Córdoba, Colombia. The same letters indicate no significant differences.

árboles no hubo diferencias (Fisher, $\mathrm{p}<0.05$ ). La mayor abundancia de aves se registró en el BS con el $38 \%$ de los individuos, seguido de PAC con $28 \%$, PBC con $21 \%$ y Po con $13 \%$. Se presentaron diferencias significativas en la abundancia de especies entre estos hábitats (Fisher, $p=0.0007$ ) (Fig. 2). La abundancia del hábitat de $\mathrm{BS}$ fue diferente de los hábitats de PBC y Po, mientras que PAC no presentó diferencias con $\mathrm{PBC}$ ni con el BS.

La diversidad de aves fue mayor en BS según el índice de Shannon, en contraste se presentó mayor dominancia de especies en los hábitats de potreros según el índice de Simpson (Cuadro 1). Estos índices miden el grado de heterogeneidad que hay en la complejidad del hábitat y repartición de los recursos: el hábitat de bosque resultó ser el que presentó mayor heterogeneidad y equilibrio en la distribución y oferta de recursos, ya que presentó a su vez una baja dominancia de especies.

Dinámica espacial y temporal: Se registró la mayor riqueza de especies en octubre y noviembre, mientras que en los otros meses se 
CUADRO 1

Índices de diversidad para la avifauna registrada en cuatro hábitats en el Departamento de Córdoba, Colombia

TABLE 1

Diversity indices for birds recorded in four habitats in the Department of Córdoba, Colombia

\begin{tabular}{lcccc} 
& BS & PAC & PBC & Po \\
Riqueza especies & 135 & 116 & 101 & 63 \\
Número de individuos & 2562 & 1836 & 1392 & 877 \\
Dominancia & 0.025 & 0.029 & 0.034 & 0.095 \\
Shannon & 4.133 & 3.938 & 3.892 & 3.075 \\
\hline
\end{tabular}

*Bosque secundario (BS), potrero con alta cobertura de árboles (PAC), potrero con baja cobertura de árboles $(\mathrm{PBC})$, potrero sin árboles $(\mathrm{Po})$.

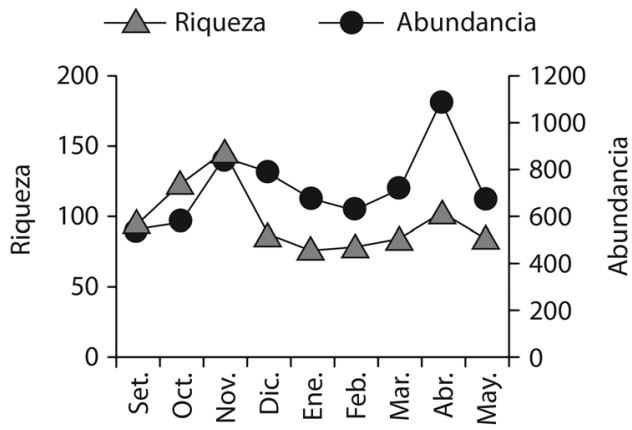

Fig. 3. Dinámica temporal de la riqueza y abundancia de aves en hábitat de bosque seco tropical en el departamento de Córdoba.

Fig. 3. Temporal dynamics the richness and abundance of birds in tropical dry forest habitats in the Department of Córdoba, Colombia.

observaron riquezas muy similares. La abundancia fue mayor en abril y noviembre, $y$ menor en septiembre (Fig. 3). En enero, febrero y marzo, hubo poca variación en la riqueza y abundancia; el pico más alto en los registros del número de individuos y número de especies se presentó en noviembre, esto se debió principalmente a la influencia de la presencia y ausencia de las aves migratorias neotropicales. El otro pico de registro de mayor abundancia y riqueza fue para abril y mayo.

Algunas especies residentes estacionales que se registraron en noviembre también contribuyeron a que se reportara la mayor riqueza en la escala temporal estudiada. Algunas especies típicas de bosques húmedos fueron registradas, lo cual es indicador de que los bosques secos tropicales también sirven de uso transicional para especies de bosques húmedos, pues en noviembre se registraron especies únicas que no fueron observadas en ninguna otra ocasión durante el estudio y que además son muy raras en ecosistemas de bosque seco tropical.

Los hábitats estudiados presentaron tendencias a distanciarse en cuanto a la distribución de las especies presentes, el hábitat Po presentó la mayor distancia en comparación a los hábitats con cobertura arbórea, los cuales compartieron gran proporción de las especies registradas. El análisis de correspondencia sin tendencia (DCA) muestra la distribución de todas las especies de aves entre los hábitats, este explica el $75 \%$ de la variabilidad de los datos en sus dos primeros ejes (Fig. 4). Los círculos en el grafico corresponden a las elipses de confianza al $95 \%$, con lo cual se muestra la alta diferencia que hay en las especies compartidas entre Po y los hábitats con cobertura arbórea. La distribución de los hábitats en el primer eje del grafico muestra que hay una posible transición entre Po y el BS estando en las zonas intermedias los hábitats de $\mathrm{PBC}$ y PAC, entre los cuales se observan en el gráfico muchas unidades entremezcladas, lo que hace suponer una alta similitud entre sus ensamblajes de aves.

\section{DISCUSIÓN}

Debido a las características del paisaje la familia de los Tyrannidae fue dominante, ya que son aves en su mayoría de hábitos insectívoros, muy adaptables tanto a zonas bien conservadas como a áreas abiertas intervenidas. Otros, autores como Cárdenas et al., (2003) y Vilchez et al., (2008) las han catalogado como especies características de hábitats abiertos $\mathrm{y}$ agropaisajes fragmentados. Esto las hace un grupo de aves muy común en los bordes de bosque, claros y potreros arbolados, por lo cual están bien representadas, y son dominantes en 


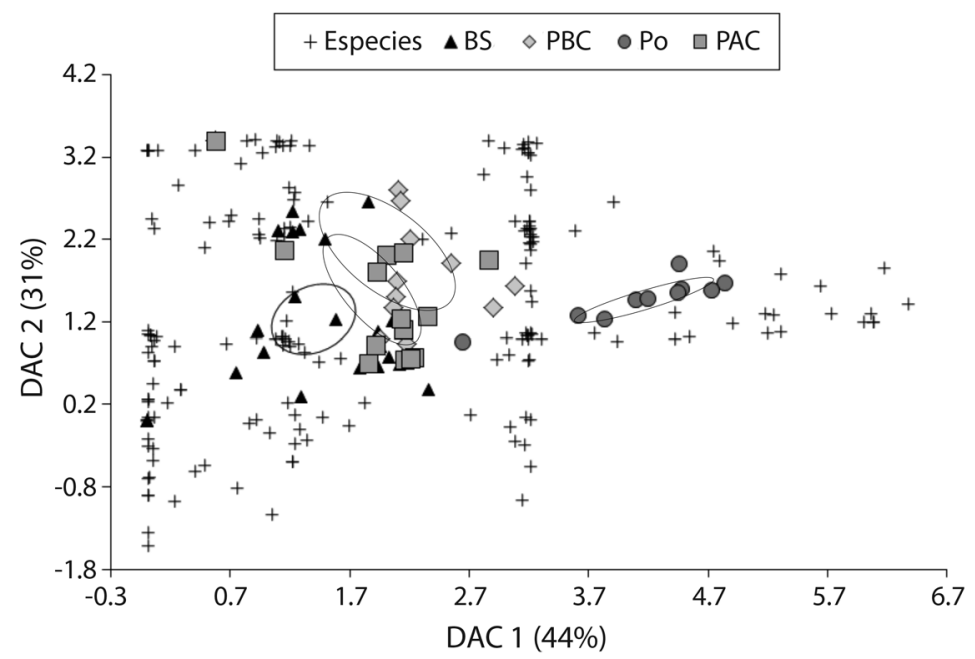

Fig. 4. Análisis de correspondencia sin tendencia (DCA) para las especies de aves presentes en cuatro hábitats en paisajes ganaderos del Departamento de Córdoba, Colombia.

Fig. 4. Detrended correspondence analysis (DCA) for bird species in habitats uses in cattle landscapes of the Department of Córdoba, Colombia.

casi todos los hábitats disponibles de la región del Departamento de Córdoba.

En países como Costa Rica, Nicaragua y Colombia, se han reportado impactos considerables de la cobertura arbórea en paisajes fragmentados dominados por ganadería extensiva, resaltando a los sistemas silvopastoriles, ya que favorecen la riqueza y abundancia de aves en paisajes ganaderos (Sáenz, Villatoro, Ibrahim, Fajardo, \& Pérez, 2006). Estos mismos autores reportan en Rio la Vieja (Colombia) riquezas sin diferencias significativas entre sistemas silvopastoriles y parches de bosques secundarios. Otros estudios también han reportado que no encontraron diferencias significativas entre sistemas agroforestales y áreas de bosques, sin embargo, se debe tener en cuenta que las diferencias importantes entre los tipos de hábitats, radican más en la composición de especies presentes y las proporciones de especies que comparten. Así nuestros resultados concuerdan con los de Harvey et al. (2006b) y Vilchez et al. (2008) ya que sugieren que las diferencias más notables entre los hábitats no son el número de especies ni las abundancias presentes, sino cuáles especies están presentes en cada uso de suelo.
En Nicaragua, Vílchez et al. (2008) determinaron que la diversidad de árboles es la variable que mejor discrimina la variación de la diversidad de aves en diferentes hábitats en un paisaje fragmentado por ganadería. Por su parte, Sáenz et al. (2006) encontraron alta correlación entre la riqueza de aves, y la riqueza y cobertura de la vegetación, siendo la cobertura del dosel la variable que mejor explica la alta riqueza de aves en un paisaje fragmentado en Matiguás, Nicaragua. Se ha evidenciado que en los agropaisajes fragmentados, la cobertura y la heterogeneidad estructural de la vegetación, juegan un papel importante en la composición de aves presentes, influyendo en la supervivencia de las especies dependientes de la cobertura arbórea (Heikkinen, Luoto, Virkkala, \& Rainio, 2004; Sekercioglu, 2002). Bishop y Myers (2005) encontraron también que la riqueza de gremios tróficos de aves en zonas templadas, se ve muy afectada por las características de la vegetación, principalmente el porcentaje de cobertura de dosel en agropaisajes.

Es conocido que los árboles proveen alimento para las aves de bosque y otros animales, por lo tanto, es de esperarse encontrar mayor diversidad de aves en los sistemas con mayor 
complejidad en la estructura de la vegetación (Harvey, Guindon, Haber, DeRosier, \& Murray, 2008). Cárdenas et al. (2003) encontraron que en potreros de alta cobertura de árboles y bosques ribereños, hubo más diversidad según el índice de Shannon, comparados con fragmentos de bosque seco y potreros de baja cobertura en paisajes fragmentados en Cañas-Costa Rica. Este estudio sugiere que los fragmentos de hábitats boscosos son importantes dentro del paisaje, debido a que pueden conservan comunidades de aves propias de bosques, las cuales no se mantendrían en los hábitats abiertos. Sin embargo, también es importante tener en cuenta los hábitats abiertos con árboles, como parte de las estrategias de conservación (Cárdenas et al., 2003; Harvey et al., 2006b; Harvey, González, \& Somarriba, 2006a; Vilchez et al., 2014) ya que estos contribuyen a la conservación por medio de la conectividad estructural que generan en el paisaje, lo cual permite la movilidad de algunas especies tolerantes a los hábitats intervenidos o que dependen del bosque (Chacón \& Harvey, 2008; Vergara, 2015).

La dinámica temporal del ensamblaje de aves en los hábitats estudiados estuvo muy influenciada por especies migratorias, ya que entre los meses de octubre y noviembre se registra la mayor llegada de migratorias neotropicales a Colombia, que corresponde a la migración de otoño (Bayly, Cárdenas-Ortiz, Rubio, \& Gómez, 2014). En los meses de abril y mayo se da la migración de primavera época en la cual se presentó el otro pico de mayor registro de individuos y especies en este estudio. Esta migración ocurre con el paso de muchos individuos que vienen del sur del continente y que utilizan los parches de bosque en el Caribe colombiano como sitio de descanso en su viaje migratorio, lo que se refleja en el alto número de individuos registrados en abril. El tránsito de las aves migratorias también es una razón de peso para resaltar el valor de conservación que juegan los agropaisajes para la biodiversidad neotropical, ya que son muchas las especies de aves migratorias que dependen de la cobertura arbórea, donde los sistemas silvopastoriles pueden servir como sitio de descanso y de recarga energética, para estas especies en su viaje de migración (DíazBohórquez, Bayly, Botero, \& Gómez, 2014; Vergara, 2009, 2015).

Durante los meses de noviembre a marzo, la especie más abundante fue Setophaga petechia, esta es una especie migratoria neotropical que permanece en el norte de Suramérica entre los meses que coinciden con la época seca en el Departamento de Córdoba (BirdLife International, 2006). En Colombia S. petechia es abundante durante toda la temporada de migración, y hay registros de que algunos pocos individuos ingresan al país a mediados de agosto. Las mayores oleadas migratorias se destacan a lo largo de la costa del Caribe, en las cuales llegan varias especies, estableciéndose principalmente en los bosques secos y húmedos en tierras bajas (American Ornithologists' Union, 1998; Díaz-Bohórquez et al., 2014; Gómez et al., 2011; Hilty \& Brown, 1986; Restall et al., 2006; Stiles, 2004).

El análisis global de la distribución de especies y la composición dentro de un mosaico de paisaje, permite entender el aporte y el papel que juegan cada uno de los hábitats disponibles en la distribución de las especies y la capacidad de estos para refugiarlas y garantizar su conservación en paisajes intervenidos. El mosaico del paisaje del Departamento Córdoba está dominado por potreros con diferentes densidades de árboles dispersos, pero en su mayoría son áreas de ganadería extensiva desprovistos de árboles, y se mantiene la cobertura solo en algunas cercas vivas y fragmentos de bosque secundario. Estos fragmentos de bosque se pueden convertir en muchos casos en un refugio importante para las especies dependientes de bosque que aún habitan en el paisaje, lo que representa una gran opción, que puede permitir su mayor supervivencia, ya que muchas son poco tolerantes a las áreas abiertas intervenidas (Mendoza, Jimenez, Lozano-Zambrano, Renjifo, \& Caicedo-Rosales, 2008). Muchas de las especies dependientes de la cobertura forestal y que también se encuentran en áreas abiertas, son de los animales que más aportan servicios ecosistémicos importantes para los sistemas 
productivos, por lo cual, se convierten en especies claves desde el punto de vista funcional en estos hábitats (Petit \& Petit, 2003).

\section{AGRADECIMIENTOS}

A la Universidad de Córdoba y al grupo de investigación Biodiversidad Unicórdoba por la financiación de esta investigación en el marco del proyecto "Estudio de la composición de la fauna silvestre asociada a las reservas naturales de la sociedad civil en el departamento de Córdoba-Colombia". A COLCIENCIAS y al Programa Jóvenes Investigadores e Innovadores "Virginia Gutiérrez de Pineda". A Valentín Espitia, Leidys Murillo y Luis Morelo por el apoyo en el trabajo campo.

\section{RESUMEN}

En Colombia el bosque seco tropical es una de los ecosistemas más amenazados y menos conocidos del país. Se desconoce mucho cómo los procesos de transformación y fragmentación de hábitat han afectado las condiciones y supervivencia de la avifauna del bosque seco. Este trabajo evaluó la influencia de los elementos del paisaje (bosques y potreros de producción ganadera con diferentes densidades de árboles) en la diversidad de aves en la región del bosque seco tropical del Departamento de Córdoba, Colombia. El trabajo se desarrolló caracterizando la avifauna en cuatro localidades conformadas por fragmentos de bosque secundario dentro de un paisaje dominado por áreas de ganadería extensiva. Se identificaron cuatro tipos de hábitats en el paisaje según la densidad de cobertura arbórea: fragmentos de bosque secundario, potreros con alta cobertura de árboles, potreros con baja cobertura árboles y potreros sin árboles. Con el fin de evaluar la influencia de la cobertura arbórea en la diversidad de aves, se realizaron muestreos en transectos de observación durante los meses de septiembre 2011 a mayo 2012 con muestreos mensuales. Los transecto tuvieron $200 \mathrm{~m}$ de longitud para el conteo de aves en cada hábitat, las observaciones se hicieron en la mañana (6:00 - 9:00) y en la tarde (15:00 - 18:00). Se registraron 6667 individuos pertenecientes a 180 especies y 48 familias de aves. Las especies más abundantes fueron: Columbina talpacoti (5.37\%), Brotogeris jugularis (5.14\%), Tyrannus melancholicus (4.81\%) y Bubulcus ibis (4.80\%). La familia con mayor abundancia fue Tyrannidae (16.4\%), seguida de Psitacidae (9.0\%) y Columbidae (8.2\%). La familia que presentó mayor número de especies fue: Tyrannidae con 28 especies, seguida de Accipitridae e Icteridae con 10 especies cada una. La mayor riqueza de especies se presentó en el hábitat de bosque secundario, seguido de los potreros con alta cobertura de árboles y potreros con baja cobertura de árboles; la menor abundancia y riqueza de especies se encontró en los potreros sin árboles, presentándose diferencias significativas entre los hábitats (Fisher, $\mathrm{p}<0.05$ ). La diversidad de aves fue mayor en los bosques secundarios según el índice de Shannon, en contraste, la menor diversidad se encontró en los potreros sin árboles. La mayor riqueza de especies se presentó en octubre y noviembre; en el resto del período de muestreo hubo estabilidad en el número de especies e individuos registrados. Se evidenció la importancia de la presencia de árboles en áreas de ganadería extensiva, debido a que ayudan a contener una importante diversidad de especies, así como a generar conectividad con los fragmentos de bosques secundarios presentes en el paisaje ganadero.

Palabras claves: aves, región caribe, biodiversidad, sistemas silvopastoriles, paisajes ganaderos.

\section{REFERENCIAS}

American Ornithologists' Union. (1998). Check-list of North American birds: the species of birds of North America from the Arctic through Panama, including the West Indies and Hawaiian Islands (7 ed.) (7th edition). Lawrence, Kansas, USA: American Ornithologists' Union.

Ballesteros, J., Vergara, J., \& Linares, J. C. (2015). Aves de Córdoba. En J. Ballesteros \& J. C. Linares (Eds.), Fauna de Córdoba, Colombia (p. 324). Monteria, Colombia: Grupo de Investigación Biodiversidad Unicórdoba, Universidad de Córdoba.

Bayly, N. J., Cárdenas-Ortiz, L., Rubio, M., \& Gómez, C. (2014). Migration of raptors, swallows and other diurnal migratory birds through the Darien of Colombia. Ornitología Neotropical, 25(May), 63-71.

BirdLife International. (2006). Conservando las Aves Migratorias Neotropicales en los Andes Tropicales. Quito, Ecuador: BirdLife International y U.S. Fish and Wildlife Service.

Bishop, J. A., \& Myers, W. L. (2005). Associations between avian functional guild response and regional landscape properties for conservation planning. Ecological Indicators, 5(1), 33-48.

Buckland, S. T., Anderson, D. R., Burnham, K. P., \& Laake, J. L. (2005). Distance Sampling. En P. Armitage \& T. Colton (Eds.), Encyclopedia of Biostatistics (2nd ed., pp. 1-6). USA: John Wiley \& Sons, Ltd.

Cárdenas, G., Harvey, C. A., Ibrahim, M., \& Finegan, B. (2003). Diversidad y riqueza de aves en diferentes hábitats en un paisaje fragmentado en Cañas, Costa Rica. Agroforestería en Las Américas, 10, 78-85.

Chacón, M., \& Harvey, C. A. (2008). Contribuciones de las cercas vivas a la estructura y la conectividad de 
un paisaje fragmentado en Rio Frio, Costa Rica. En C. A. Harvey \& J. C. Sáenz (Eds.), Evaluación $y$ conservación de biodiversidad en paisajes fragmentados de Mesoamérica (pp. 225-248). Santo Domingo de Heredia, Costa Rica: Instituto Nacional de Biodiversidad (INBio).

Chao, A. (1984). Nonparametric Estimation of the Number of Classes. Scandinavian Journal of Statistics, 11, 265-270.

Colwell, R. K. (2013). EstimateS: Statistical Estimation of Species Richness and Shared Species from Samples, Versión 9.1.0. University of Connecticut, USA. Retrieved from http://viceroy.eeb.uconn.edu/ estimates/

Di Rienzo, J. A., Casanoves, F., Balzarini, M. G., Gonzalez, L., Tablada, E. M., \& Robledo, C. W. (2014). Infostat versión 2014. Córdoba, Argentina: Grupo Infostat, FCA, Universidad Nacional de Córdoba. Retrieved from www.infostat.com.ar

Di Rienzo, J. A., Casanoves, F., Pla, L., Vilchez, S., \& Di Rienzo, M. J. (2010). Qeco-Quantitative ecology software: A collaborative approach. Revista Latinoamericana de Conservación, 1(1), 73-75.

Díaz-Bohórquez, A., Bayly, N. J., Botero, J. E., \& Gómez, C. (2014). Aves migratorias en agroecosistemas del norte de Latinoamérica, con énfasis en Colombia. Ornitología Colombiana, 14(November), 3-27.

Donegan, T., McMullan, W. M., Quevedo, A., \& Salaman, P. (2013). Revision of the status of bird species occurring or reported in Colombia 2013. Conservación Colombiana, 19, 3-10.

Gillespie, T. W., \& Walter, H. (2001). Distribution of bird species richness at a regional scale in tropical dry forest of Central America. Journal of Biogeography, 28(5), 651-662.

Gómez, C., Bayly, N., González, A., Abril, E., Arango, C., Giraldo, J., ... Naranjo, A. (2011). Avances en la investigación sobre aves migratorias neárticasneotropicales en Colombia y retos para el futuro: trabajos del III Congreso de Ornitología Colombiana. Ornitología Colombiana, 11, 3-13.

Gomez, J. P., \& Robinson, S. K. (2014). Aves del bosque seco tropical de Colombia: Las comunidades del Valle Alto del Río Magdalena. En C. Pizano \& H. Garcia (Eds.), El Bosque Seco Tropical en Colombia (pp. 95-127). Bogotá D.C., Colombia: Instituto de Investigación de Recursos Biologicos Alexander von Humboldt (IAvH).

Harvey, C. A., González, J., \& Somarriba, E. (2006). Dung beetle and terrestrial mammal diversity in forests, indigenous agroforestry systems and plantain monocultures in Talamanca, Costa Rica. Biodiversity and Conservation, 15(2), 555-585.
Harvey, C. A., Guindon, C. F., Haber, W. A., DeRosier, D. H., \& Murray, K. G. (2008). Importancia de los fragmentos de bosque, los árboles dispersos y las cortinas rompevientos para la biodiversidad local y regional: El caso de Monteverde, Costa Rica. En C. A. Harvey \& J. C. Sáenz (Eds.), Evaluación y conservación de biodiversidad en paisajes fragmentados de Mesoamérica (pp. 289-325). Santo Domingo de Heredia, Costa Rica: Instituto Nacional de Biodiversidad (INBio).

Harvey, C. A., Medina, A., Sánchez, D., Vilchez, S., Hernández, B., Sáenz, J. C., ... Sinclair, F. L. (2006). Patterns of animal diversity in different forms of tree cover in agricultural landscapes. Ecological Applications, 16(5), 1986-1999.

Heikkinen, R. K., Luoto, M., Virkkala, R., \& Rainio, K. (2004). Effects of habitat cover, landscape structure and spatial variables on the abundance of birds in an agricultural-forest mosaic. Journal of Applied Ecology, 41(5), 824-835.

Hill, M. O., \& Gauch, H. G. (1980). Detrended correspondence analysis: An improved ordination technique. Vegetatio, 42, 47-58.

Hilty, S. L., \& Brown, W. L. (1986). A guide to the birds of Colombia. Princeton, NJ.: Princeton University Press.

Krebs, C. J. (1999). Ecological methodology (second edition). Menlo Park, California USA: Benjamin/Cummings.

Legendre, P., \& Legendre, L. (1998). Numerical Ecology (Second Eng). Amsterdam: Elsevier Science B.V.

Magurran, A. E. (2004). Measuring Biological Diversity. Oxford, UK: Blackwell Publishing.

Mendoza, J., Jimenez, E., Lozano-Zambrano, F., Renjifo, L. M., \& Caicedo-Rosales, P. (2008). Identificación de elementos del paisaje prioritarios para la conservación de biodiversidad en paisajes rurales de los andes centrales de Colombia. En C. A. Harvey \& J. C. Sáenz (Eds.), Evaluación y conservación de biodiversidad en paisajes fragmentados de Mesoamérica 2 (pp. 252-288). Santo Domingo de Heredia, Costa Rica: Instituto Nacional de Biodiversidad (INBio).

Murgueitio, E., Ibrahim, M., Ramirez, E., Zapata, A., Mejia, C. E., \& Casasola, F. (2003). Usos de la tierra en fincas ganaders: Guía para el pago de servicios ambientales en el proyecto Enfoques Silvopastoriles Integrados para el Manejo de Ecosistemas. Medellin, Colombia: Fundación CIPAV, CATIE, UCA- NITLAPAN.

Ojasti, J. (2000). Manejo de Fauna Silvestre Neotropical (F. Dallmeier, Ed.). Washington D.C.: Smithsonian Intitution.

Oksanen, J. (2013). Multivariate Analysis of Ecological Communities in $R$ : vegan tutorial. Retrieved 
from http://cc.oulu.fi/ jarioksa/opetus/metodi/vegantutor.pdf

Oksanen, J., Kindt, R., Legendre, P., O’Hara, B., Simpson, G. L., Solymos, P., ... Wagner, H. (2009). The Vegan Package: Communiity Ecology Package. Retrieved from http://cran.r-project.org/, http://vegan.r-forge.rproject.org/

Petit, L. J., \& Petit, D. R. (2003). Evaluating the importance of human-modified lands for Neotropical bird conservation. Conservation Biology, 17(3), 687-694.

Pizano, C., Cabrera, M., \& Garcia, H. (2014). Bosque seco tropical en Colombia; generalidades y contexto. En C. Pizano \& H. Garcia (Eds.), El Bosque Seco Tropical en Colombia (pp. 37-47). Bogotá D.C., Colombia: Instituto de Investigación de Recursos Biologicos Alexander von Humboldt (IAvH).

Ralph, C. J., Geupel, G. R., Pyle, P., Martin, T. E., DeSante, D. F., \& Milá, B. (1996). Manual de métodos de campo para el monitoreo de aves terrestres (General Technical Report). Albany, California: Pacific Southwest Research Station, Forest Service, U.S. Department of Agriculture.

Restall, R., Rodner, C., \& Lentino, M. (2006). Birds of Northern South America: An Identification Guide: Plates and Maps. New Haven: Yale University Press.

Rodríguez, G., Banda-R, K., Reyes, S., \& Estupiñán, A. (2012). Lista comentada de las plantas vasculares de bosques secos prioritarios para la conservación en los departamentos de Atlántico y Bolívar (Caribe colombiano). Biota Colombiana, 13(2), 7-39.

Sáenz, J. C., Villatoro, F., Ibrahim, M., Fajardo, D., \& Pérez, M. (2006). Relación entre las comunidades de aves y la vegetación en agropaisajes dominados por la ganadería en Costa Rica, Nicaragua y Colombia. Agroforestería En Las Américas, 45, 37-48.
Schroth, G., da Fonseca, G., Harvey, C. A., Gascon, C., Vasconcelos, H. L., \& Izac, A. M. (Eds.). (2004). Agroforestry and biodiversity conservation in tropical landscape. Washinton D.C.: Island Press.

Sekercioglu, C. H. (2002). Effects of forestry practices on vegetation structure and bird community of Kibale National Park, Uganda. Biological Conservation, 107(2), 229-240.

Stiles, F. G. (2004). Austral migration in colombia: the state of knowledge, and suggestions for action. Ornitología Neotropical, 15(Suplemento), 349-355.

Vergara, J. (2009). Avifauna presente en sistemas silvopastoriles con diferentes arreglos vegetales en Corpoica centro de investigación Turipaná, Córdoba - Colombia (Tesis de pregrado). Universidad de Córdoba, Montería, Colombia.

Vergara, J. (2015). Caracterización de la degradación y los cambios de usos de suelo en fincas ganaderas y su relación con la diversidad de aves en el Valle del Rio Cesar, Colombia (Tesis de Maestría). Centro Agronomico Tropical de Investigación y Enseñanza (CATIE), Turrialba, Costa Rica.

Vilchez, S., Harvey, C. A., Sáenz, J. C., Casanoves, F., Carvajal, J. P., Villalobos, J., ... Sinclair, F. L. (2014). Consistency in bird use of tree cover across tropical agricultural landscapes. Ecological Applications, 24(1), 158-168.

Vilchez, S., Harvey, C. A., Sanchez, D., Medina, A., Hernandez, B., \& Taylor, R. (2008). Diversidad y composición de aves en un agropaisaje de Nicaragua. En C. A. Harvey \& J. C. Sáenz (Eds.), Evaluación $y$ conservación de biodiversidad en paisajes fragmentados de Mesoamérica (pp. 547-576). Santo Domingo de Heredia, Costa Rica: Instituto Nacional de Biodiversidad (INBio). 